\title{
Impacto de los sistemas de salud y educación en la vivencia de una madre con un niño con síndrome de Down
}

RESUMEN: Los niños con síndrome de Down presentan diversos problemas de salud, del comportamiento y del aprendizaje que requieren atención especial. Debido a ello tienen dificultades de recibir atención adecuada. De consecuencia formas de ineficiencia del sistema sanitario y educativo impactan a nivel personal y familiar. Sin embargo, en Perú existe un vacío de información de naturaleza empírica y cualitativa sobre este efecto en la vida de estos niños y sus padres. Empleando un análisis cualitativo de los contenidos de una entrevista a una madre se identificaron siete temas vinculados al impacto de estos dos sistemas: orientación y apoyo, ámbito laboral y condición de salud, relación con el sistema de salud, relación con la escuela, discriminación hacia las personas con discapacidad, cambio de perspectiva respecto a la discapacidad y comunicación no convencional. Los resultados evidencian que las formas de ineficiencia de los sistemas sanitario y educativo impactan negativamente en la vida de las personas con discapacidad y en sus familias. Este impacto afecta la vida familiar y laboral determinado las decisiones educativas $y$ de salud. Igualmente, persisten todavía formas de no aceptación y modalidades de discriminación más o menos evidentes, no obstante los esfuerzos por la inclusión. Las actitudes negativas hacia la discapacidad pueden ser condicionadas por su severidad. De hecho, la aceptación de la discapacidad es un proceso en el que se modifica la percepción. En el mismo, es fundamental enfocarse en la persona y no simplemente en los límites de su condición.

PALABRAS CLAVE: Discapacidad intelectual; Síndrome de Down, Familia; Sistemas de salud; Análisis cualitativo; Perú.

Citar como: Enríquez Y. Impacto de los sistemas de salud y educación en la vivencia de una madre con un niño con síndrome de Down. CASUS. 2017;2(3):169-178. 


\section{INTRODUCCIÓN}

El síndrome de Down (SD) es la anomalía cromosómica más común caracterizada por una disfunción del desarrollo (1). El mismo es una de las principales causas de discapacidad intelectual (DI) asociándose a diversos problemas de salud, entre ellos problemas cardíacos, distrofia muscular y epilepsia (1). La Organización Mundial de la Salud estima que 1 de 1100 nacidos vivos (NV) presenta este desorden cromosómico (2). Aunque es escasa la información sobre la incidencia del SD para la población peruana, para el 2013 un estudio de incidencia hospitalaria reportó que 5.1 de cada $1000 \mathrm{NV}$ fueron diagnosticados con esta anomalía en Lima (3).

Se considera que los niños con SD tienen necesidades especiales de atención médica (4). Igualmente presentan un incremento del riesgo de afecciones crónicas, por lo que requieren mayores servicios de salud respecto a los niños en general $(4,5)$. Añadidos a los problemas de salud mencionados tienen un cociente de inteligencia bajo y presentan dificultades en el cálculo, la escritura y la lectura (6-8). Por otro lado, evidencian problemas a nivel conductual o emocional $(6,9)$. Para los niños con SD estas dificultades pueden tener un valor acumulativo.

Los problemas que pueden experimentar consecuentemente reducen su calidad de vida así como la de sus familias $(10,11)$. Debido a las complicadas situaciones que enfrentan tienen dificultades en recibir adecuada atención, tanto a nivel médico cuanto educativo $(10,12,13)$. Por otro lado, a nivel social muchas personas con SD se sienten aisladas, indeseadas o perciben ser una carga para la sociedad (14). Igualmente, sus familias pueden enfrentar actitudes negativas, experimentando pobreza y exclusión social. Muchos padres de niños con SD sienten que pasan demasiado tiempo luchando contra la burocracia (14). Como es de esperar las formas de ineficiencia de los sistemas sanitarios y educativos tienen un impacto negativo sea a nivel personal como familiar.

Por ejemplo, se ha indagado en este efecto a través de las experiencias de pacientes con SD y en sus familias reportándose discriminación a motivo de su condición $(15,16)$. Estos modos de discriminación incluyen formas indirectas vinculadas a la no consideración de sus necesidades por el personal sanitario y la no satisfacción de las mismas por los servicios de salud
(16). Otras vivencias indican procesos engorrosos para la obtención de servicios de salud mental o dificultad en la comprensión de los modos para obtener ayuda encontrando numerosos obstáculos (17).

No obstante la profundización de este tema en otros países $(4,5,15-17)$, sin embargo en América Latina y en Perú, continúa sin estudiarse en modo profundo este impacto. Un vacío de información que se evidencia en los escasos estudios de naturaleza empírica y cualitativa que indagan sobre el efecto en la vida de los niños con SD y sus padres. Teniendo en consideración estos elementos se buscó examinar cualitativamente la experiencia de una madre de un niño con SD explorando sus vivencias en la aceptación de la condición de discapacidad y en cómo diversas formas de ineficiencia han incidido en las decisiones de salud y educativas de su hijo.

\section{MATERIALES Y MÉTODOS}

Estudio cualitativo de análisis de un caso. Para la investigación la participante fue captada en una institución educativa especial de Lima. La recolección de datos se realizó mediante una entrevista personal. Para el estudio se identificó previamente un conjunto claro de temas. Se empleó para recolectar información una guía estructurada de preguntas utilizando un enfoque abierto y no directivo (18). La entrevista permitió y alentó a la participante a debatir sobre otros temas relevantes, que no necesariamente se encontraban presentes en la guía de la entrevista (19).

Bajo el consentimiento de la participante se grabó el audio de la entrevista. Posteriormente se analizaron las transcripciones de la misma. Se utilizó el análisis temático descrito por Braun y Clarke (20). El marco de codificación se desarrolló en modo gradual mediante la lectura y re-lectura de las transcripciones hasta diseñar un conjunto final de temas de los datos recolectados. El procedimiento incluyó la codificación sistemática e inductiva considerando la recopilación de los datos relevantes para el objetivo del estudio y la posterior codificación en temas potenciales.

Paralelamente a la obtención del consentimiento informado se garantizaron el anonimato de la participante y la confidencialidad de los datos recolectados. Igualmente se aseguró la confidencialidad de la información durante el proceso de análisis empleando seudónimos para las personas o instituciones involucradas en la transcripción y en la difusión de los resultados. 


\section{RESULTADOS}

Características de la encuestada

Participó en este estudio una madre peruana con educación superior, de 49 años que se encontraba casada al momento de la entrevista. Cuando se recopilaron los datos ella trabajaba a tiempo parcial y su esposo a tiempo completo. La entrevistada es madre de tres niños entre los nueve y los catorce años. El segundo de sus hijos (doce años) presenta el SD. El mismo asiste a un centro de educación básica especial de la ciudad de Lima.

\section{Principales temas}

El análisis de las transcripciones reportó siete temas principales: orientación y apoyo, ámbito laboral y condición de salud, relación con el sistema de salud, relación con la escuela, discriminación hacia las personas con discapacidad, cambio de perspectiva respecto a la discapacidad y comunicación no convencional (ver tabla 1). Seguidamente los temas se detallan empleando citas de la entrevistada. solo un psicólogo del hospital me dio su tarjeta para una visita privada.",

Ámbito laboral y condición de salud

La entrevistada igualmente habló sobre la dificultad en compatibilizar la vida laboral y las necesidades vinculadas a la condición de salud de su hijo. La participante señaló que esta dificultad en la armonización de los dos ámbitos se conecta al mayor requerimiento de tiempo para poder responder a las necesidades de salud de su hijo. De este modo, aún disponiendo de un tiempo parcial, este resultó insuficiente:

"Todavía estaba trabajando, pero después de unos meses me di cuenta de que ya no me lo podía permitir. Me habían concedido el tiempo parcial, pero no fue suficiente para acompañarlo a todas las visitas y tratamientos."

\section{Tabla 1. Resumen de los principales temas y subtemas}

\begin{tabular}{l} 
Temas \\
\hline 1. Orientación y apoyo \\
2. Ambito laboral y condición de salud \\
3. Relación con el sistema de salud
\end{tabular}

\section{Relación con la escuela}

\section{Discriminación hacia las personas con discapacidad \\ 6. Cambio de perspectiva respecto a la discapacidad \\ 7. Comunicación no convencional}

\subsection{Características de la escuela \\ 4.2 Abordaje educativo e información \\ 4.3 Consecuencias existenciales \\ 4.1 Oportunidades de la escuela}

Orientación y apoyo

El primer tema identificado fue la necesidad de orientación y apoyo que se experimenta cuando se recibe el diagnóstico de SD. Paralelamente se señaló una carencia de atención adecuada a esta necesidad en las estructuras sanitarias:

\footnotetext{
"Para Ángel y para nuestra familia el camino ha ido siempre cuesta arriba: a partir de ese día, en el hospital..., en que se nos dijo que su composición genética era diferente, "especial", como se dice ahora. Él es el hijo del medio, y tan pronto como el equipo del hospital me informó me sentí perdida. Nadie te prepara, ninguno te acoge:
}

Relación con el Sistema de salud

Respecto a las características de la condición de vida de su hijo la entrevistada manifestó algunas falencias en las respuestas del Sistema de salud. En modo particular, mencionó un déficit relacionado a dos aspectos: en otorgar soporte para la realización de terapias físicas y del lenguaje, así como en la realización de diagnósticos certeros. En la familia estos dos elementos desembocaron en dos decisiones conectadas entre sí. Por un lado, se decidió suplir económicamente estas terapias de rehabilitación. Por el otro, dar seguimiento a las mismas teniendo como consecuencia el prescindir de una fuente de ingresos: 
"Ángel comenzó a caminar a los 18 meses, y el seguro de salud indicó interrumpir la fisioterapia. Pero él aún tenía muchos movimientos que aprender, y empecé a ocuparme yo, acompañándolo y pagando atenciones privadas.

Luego nos dijeron que Ángel era autista, porque no hablaba, por lo que también le quitaron el el apoyo del terapeuta del lenguaje. Pero yo pedí tres distintos diagnósticos de tres diferentes hospitales públicos y todos me confirmaron que era un niño vivaz, curioso, inteligente. Así que también continuamos pagando la terapia del lenguaje."

\section{Relación con la escuela}

El cuarto tema identificado fue la relación con la escuela. La entrevistada refirió la no eficiencia de la escuela en el abordaje adecuado de las necesidades educativas de su hijo. El argumento se subdividió en cuatro subtemas: (1) características de la escuela, (2) abordaje educativo e información, (3) consecuencias existenciales y (4) oportunidades de la escuela.

\section{Características de la escuela}

La entrevistada identificó algunas características propias de la organización y funcionamiento escolar que no facilitaron el desarrollo de su hijo a nivel educativo. Por un lado mencionó la continua rotación de profesores debida a ausencias y cambios. Esta situación tuvo como consecuencia dificultades en la adaptación del niño a la nueva circunstancia:

\footnotetext{
"Doce años y la mitad de ellos los ha pasado a acostumbrarse a un maestro diferente: todos los años una despedida, una renuncia, cada mes de marzo recomenzar todo de nuevo. (...) Cada año, es lo mismo en la escuela: ausencias y cambios de los profesores..."
}

Por otro lado, la entrevistada refirió que, en parte, el proceso de enseñanza-aprendizaje de su hijo ha resultado ineficiente debido al número excesivo de estudiantes en los grupos donde ha participado. Se debe contextualizar que en el sistema educativo nacional es frecuente que en la misma aula compartan horas de clases niños con diferentes discapacidades cognitivas:

"Al principio me decian que estarian pendientes de él, le enseñarían hasta donde pudiera aprender y reforzarían sus habilidades para relacionarse. Lo cual no ocurría porque eran demasiados alumnos por aula para un solo maestro. Y, de este modo, a mi hijo le faltó lo fundamental."

\section{Abordaje educativo e información}

En la experiencia de la entrevistada los profesores no han observado las necesidades y considerado las exigencias de su hijo. Del mismo modo, mencionó la falta de información sobre algunas circunstancias y comportamientos de su hijo respecto a los cuales no recibió respuestas oportunas por parte de la institución escolar:
"Noto que los maestros de la escuela nunca han atendido a mi hijo verdaderamente y observado sus necesidades. Un día encontraron a mi hijo desnudo en el baño y luego no quería entrar a la clase. Cuando fui a hablar con la profesora ella había renunciado y no entendí lo que había sucedido. Hechos similares se han repetido varias veces."

\section{Consecuencias existenciales}

El impacto de estos dos elementos críticos ha sido sustancial. Vinculado a lo anterior la entrevistada ofreció dos ejemplos, narrando primeramente conductas de aislamiento y agresividad en su hijo. Estos episodios tuvieron como resultado el retiro temporal del niño de la institución educativa:

\section{“(...) en el cuarto grado Ángel no pudo entrar en relación con la maestra y comenzó a golpear a sus hermanos, era agresivo con sus compañeros y se aislaba, no sabíamos qué hacer. Por eso tuve que retirarlo de la escuela. Y encima me dijeron que el problema era mío."}

En la vivencia de la entrevistada emerge igualmente insatisfacción respecto a las expectativas hacia la escuela. En este sentido, el segundo ejemplo se refiere a que desde el punto de vista didáctico tiene resultados ineficaces, pues su hijo no ha desarrollado habilidades de lectura y escritura, así como tampoco ha desarrollado el habla:
“(...) la escuela desde el punto de la enseñanza no nos ha dado casi nada: Ángel hasta ahora no habla, no lee y no escribe. La escuela debe ser un aliado de la familia, y para nosotros no ha sido así."

\section{Oportunidades de la escuela}

La encuestada mencionó que, no obstante las dificultades que ha experimentado en el proceso 
educativo de su hijo, la escuela se ha manifestado como un lugar que favorece la interacción social y expresión emocional del niño:

"Apesar de las cosas negativas la escuela le ha dado a Ángel la oportunidad de reunirse con otros niños, hacer las cosas en grupo y expresarse."

Discriminación hacia las personas con discapacidad El quinto tema reportado se refiere a la experiencia de la discriminación en diferentes contextos frente a la discapacidad del niño. La entrevistada mencionó que la discriminación, en su experiencia, ha pasado por formas de no aceptación que se manifiestan en momentos sociales o de interacción. En su parecer, detrás de la promoción de la inclusión de las personas con discapacidad aún permanece una dificultad en la acogida a nivel relacional de las personas con esta condición:

\footnotetext{
"No se dice, pero todavía muchos son reacios a aceptar una persona con discapacidad. (...) Es dificil, nadie lo dice, pero también en las reuniones sociales o en las fiestas infantiles son reacios a relacionarse con niños con alguna discapacidad."
}

Igualmente, mencionó que existen otras modalidades de discriminación que pueden resultar más o menos sutiles. En la experiencia de la entrevistada hubo situaciones en las cuales se les hizo sentir fuera de contexto o no adecuados a la situación:

\begin{abstract}
"Existen muchos que piensan que los discapacitados son una carga. Otros que solo dan consejos pero no intervienen para que algo cambie de verdad. Luego están los arrogantes que nos han hecho sentir fuera de lugar."
\end{abstract}

En modo sintético en la tabla 2, desde la perspectiva de la entrevistada, se presentan los elementos centrales de cada una de las temáticas hasta ahora abordadas subdivididos en tres esferas.

Cambio de perspectiva respecto a la discapacidad En el sexto tema identificado la participante señaló en su historia personal un momento en que realizó un cambio en la percepción respecto a la condición de su hijo. Esta modificación pasó de un hijo a quien dedicar cuidados y atenciones, a la percepción del niño como sujeto con emociones y deseos propios:

“Luego entré en contacto con la Asociación y con un educador de allí, quien me dio otra perspectiva. Empecé a considerarlo como un niño con deseos, emociones, con ganas de existir, no solo como un hijo que cuidar. Gracias a ellos, mi hijo es ahora independiente, sabe ir solo a hacer deportes, se puede preparar una comida solo."

Tabla 2. Principales dificultades reportadas según ámbito

\begin{tabular}{|c|c|}
\hline Ámbito & Elementos críticos \\
\hline sar & $\begin{array}{l}\text { - carencia de atención adecuada a la } \\
\text { necesidad de información y apoyo } \\
\text { psicológico en las estructuras } \\
\text { sanitarias cuando se recibe el } \\
\text { diagnóstico del síndrome de Down. } \\
\text { falta de soporte para la realización de } \\
\text { terapias físicas y del lenguaje. } \\
\text { dificultad en la realización de } \\
\text { diagnósticos certeros. }\end{array}$ \\
\hline edu & 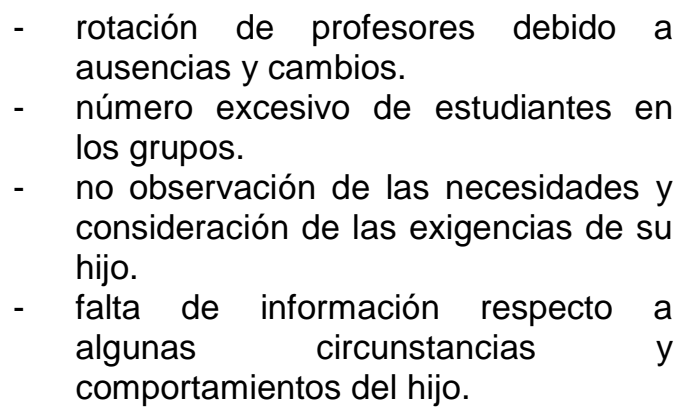 \\
\hline social & $\begin{array}{l}\text { - formas de no aceptación que se } \\
\text { manifiestan en momentos sociales o } \\
\text { de interacción. } \\
\text { modalidades de discriminación que } \\
\text { pueden resultar más o menos sutiles. }\end{array}$ \\
\hline
\end{tabular}

Paralelamente, la entrevistada señaló que el rechazo hacia las personas con SD se conecta a una mentalidad a nivel social en la que se valora positivamente la perfección. Igualmente, este modo de pensar se manifiesta en el proponer como ideal de la existencia la ausencia de dificultades y de relaciones que impliquen compromisos. No obstante, en su experiencia, la existencia de niños con discapacidad ponen en la condición de tener que cambiar de perspectiva: "Me esfuerzo por comprender a quien rechaza a
una persona con síndrome de Down. Como sucede
a veces que hay padres que abandonan a niños con
síndrome de Down y otros que no quieren 
adoptarlos. No juzgo a nadie, entiendo que ahora vivimos en la era de la perfección, nadie quiere tener molestias, inconvenientes, compromisos: nadie quiere pensar. Sin embargo los niños como Ángel obligan a cambiar de rumbo."

\section{Comunicación no convencional}

El séptimo y último tema reportado es el de la comunicación. La entrevistada manifestó que, debido a la condición de discapacidad de su hijo, ella y su familia han aprendido modos alternativos para garantizar la comunicación. La misma se manifiesta sobre todo a nivel gestual o no verbal y por encima de la comunicación oral convencional. La entrevistada señaló que esta diversidad en la comunicación no impide que sea efectiva en la transmisión de emociones. Igualmente señaló la satisfacción que le deriva:

\begin{abstract}
"Hemos aprendido a relacionarnos con él con los tonos de voz, las miradas y los gestos. No todos tenemos que expresarnos de una manera convencional, no todos somos iguales.
\end{abstract}

Su simplicidad, la felicidad de su sonrisa, totalmente espontánea: él es feliz por una mariposa, el sol, porque alguien sonríe, por los hermanos que juegan con él. Es feliz con poco, y sabe cómo hacernos felices."

\section{DISCUSIÓN}

Basándose en los hallazgos generados a partir de la entrevista se examina la percepción de una madre sobre la aceptación de la condición de discapacidad y cómo formas de ineficiencia han incidido en la salud y educación de su hijo evidenciando cómo estas impactan la vida familiar y personal. Por último, se examina la relevancia del cambio de percepción en aceptar la discapacidad y el papel que desempeña buscar otras formas de comunicación con el hijo.

Los resultados revelan falta de soporte para la realización de terapias físicas y del lenguaje, así como dificultad en la realización de diagnósticos certeros. Varios estudios han señalado que las personas con DI pueden recibir atención de salud no adecuada o se les puede negar tratamiento apropiado (10). El grupo conformado por las personas con DI, es reconocido como una población que experimenta disparidades en temas de salud (11). En este sentido, se señala que las instituciones sanitarias no siempre se encuentran preparadas o equipadas para trabajar con estas personas. Paralelamente, se reconoce la falta de conocimiento o de educación del personal de salud sobre el tratamiento a personas con SD (21). Este factor contribuye a la realización de diagnósticos errados (10). Lo cual tiene un impacto negativo a nivel personal. Cuando los síntomas que surgen de problemas de salud son atribuidos por error a la DI del individuo conduce a diagnósticos errados como a tratamientos tardíos.

Respecto al impacto de este ámbito en la familia, la entrevistada señaló la dificultad en armonizar las necesidades de salud con la vida laboral. Algunos estudios igualmente reportan que un cuarto de las familias con un hijo con necesidades especiales de salud reducen el tiempo dedicado al trabajo o lo abandonan (1, 4, 5). Este efecto negativo es particularmente relevante para las familias con hijos con SD, ya que poseen más posibilidades de visitas médicas o de ser hospitalizados respecto a otros niños (1).

La segunda área analizada fue la vinculada a la educación. La entrevista reportó factores de dificultad en el funcionamiento escolar como la rotación de profesores por ausencias y cambios. Igualmente un número excesivo de estudiantes en los grupos. Paralelamente señaló elementos negativos del comportamiento de los docentes como la no observación de las necesidades y consideración de las exigencias de su hijo y la falta de información respecto a algunos de sus comportamientos. Esta situación se revela contraproducente puesto que hallazgos indican que los niños con SD tienen un CI bajo y presentan dificultades en la memoria de trabajo, la escritura, la lectura y la aritmética (6-8). Por otro lado, estudios psicológicos reportan problemas emocionales y del comportamiento $(6,9)$. Los cambios continuos de docentes y la cantidad inadecuada de estudiantes por salón de clases pueden no garantizar adecuadas condiciones de aprendizaje (22). En este sentido, es reconocido que las dificultades a nivel de pensamiento y de comportamiento deben ser adecuadamente identificadas y tomadas en consideración puesto que limitan el proceso de enseñanza-aprendizaje (6).

Por otra parte, la entrevista arrojó la existencia de formas de no aceptación que se manifiestan en momentos sociales o de interacción. Igualmente se evidenció que subsisten modalidades de discriminación que pueden resultar más o menos sutiles, por ejemplo situaciones en las cuales se les hizo sentir fuera de contexto. En escolares son escasos 
los estudios que se han ocupado del fenómeno a nivel nacional. Sin embargo, es conocido que las personas con discapacidad identifican las actitudes negativas y las barreras sociales como el mayor impedimento para su bienestar (14), así como la razón principal de su exclusión social (11). Esas actitudes negativas y barreras sociales operan en múltiples niveles, desde lo interpersonal hasta lo social estructural $(23,24)$. Un estudio peruano reporta no solo una incapacidad cultural a valorar la diversidad de la discapacidad, sino también ideas erradas sobre la DI que limitan la inclusión (25). Por último, la diversidad de formas de no aceptación puede depender de las percepciones respecto a la permanencia y a la gravedad de la discapacidad. Ambos factores modularían la forma en que reaccionan los demás (26).

Otro hallazgo central del estudio fue la modificación en la percepción de la madre que pasó de percibirlo como un hijo a quien dedicar cuidados y atenciones, a la de un ser humano con emociones y deseos a raíz del contacto con una asociación. Otro estudio señala datos similares indicando que los padres con hijos con esta condición de vida les resultan útiles los grupos asociativos (27). En un estudio sobre el proceso de adaptación-transformación parental frente a la discapacidad de sus hijos la mayoría de los padres a raíz de la condición había alterado sus creencias en lo que respecta a los prejuicios hacia la misma (28). Igualmente, al observar a adultos con una discapacidad, aprendieron a apreciar las fortalezas y el potencial de estas personas. Desmitificar la diferencia de la discapacidad permite enfocarse en la persona y no simplemente en los límites de su condición. (28).

Por último, los resultados revelan que debido a la condición de discapacidad de su hijo, los padres y la familia han aprendido modos alternativos para garantizar la comunicación. La misma se manifiesta sobre todo a nivel gestual acompañando la comunicación oral convencional (29). La literatura reporta que en el $\mathrm{SD}$, a menudo se presentan dificultades para la comunicación verbal (30). En términos generales, son retrasos en el habla, el uso del lenguaje $\mathrm{y}$ el funcionamiento pragmático los trastornos mayormente experimentados por la personas con SD (30). Igualmente, es posible que algunos niños nunca desarrollen el habla y por ello empleen formas alternativas de comunicación (30, $31)$.
Por ello, para compensar el retraso en la producción oral varios niños con SD muestran un desarrollo significativo de la comunicación gestual, que puede variar de acuerdo con el contexto ambiental (29). Por último, los niños con SD han sido identificados por sus padres como personas que son comprendidas por el círculo de familiares y amigos (32), pero que hablan de manera ininteligible a personas ajenas al mismo o para aquellos que los encuentran por primera vez (33). La comunicación gestual compensa y es eficaz dentro de ese círculo, no obstante la dificultad experimentada.

Entre las limitaciones del estudio se señala que los resultados no son generalizables. Respecto a la calidad de la información obtenida se debe considerar que aunque la entrevistada proporcionó información en forma voluntaria, pudo haber censurado comentarios sobre lo que había o estaba experimentado. Además, el paso del tiempo y las experiencias intermedias dificultan recordar sucesos con precisión alterando las percepciones originales. Sin embargo, a través de la vivencia de la encuestada se abordó el impacto familiar de aspectos críticos en el ámbito sanitario y educativo en modo amplio y profundo.

\section{CONCLUSIÓN}

No obstante se están realizando esfuerzos por la inclusión todavía persisten formas de no aceptación y modalidades de discriminación más o menos evidentes. Las actitudes negativas hacia la discapacidad pueden ser condicionadas por la severidad de la misma. Las modalidades de discriminación se manifiestan sea a nivel interpersonal, como en lo social. Los resultados evidencian que a nivel social las formas de ineficiencia del sistema sanitario y educativo pueden tener un impacto negativo en la vida de las personas con discapacidad y en sus familias. Este impacto afecta la vida familiar y laboral determinado las decisiones educativas o de salud.

Debido a lo anterior, las instituciones de salud deben estar preparadas y equipadas para trabajar con estas personas. Paralelamente, se evidencia la necesidad de un mayor conocimiento de los proveedores de salud sobre el tratamiento a personas con SD. Paralelamente, un acceso adecuado a los servicios y la atención de salud ayudaría a las familias a mantener el empleo preservando sus fuentes de ingresos. Igualmente, los centros de educación especial deben reconsiderar elementos estructurales-organizativos propios y actitudinales del docente para garantizar una mayor 
eficacia educativa. Los profesores deben reconocer las dificultades a nivel de pensamiento y de comportamiento propias de cada patología vinculada a las diversas formas de discapacidad. Las que deben ser adecuadamente identificadas y tomadas en consideración puesto que impiden el aprendizaje

En el SD, debido a los retrasos o ausencia del habla, aprender modos de comunicación alternativos es fundamental para que los padres de familia garanticen la comunicación con sus hijos. Los mismos, manifestándose a nivel gestual acompañan la comunicación oral convencional. En este sentido constituyen modos compensatorios eficaces en la comunicación familiar.

La aceptación de la discapacidad es un proceso en el que se modifica la percepción de la madre. En este proceso parental de transformación frente a la discapacidad del hijo se modifican creencias hacia la discapacidad. En el mismo, es fundamental apreciar las fortalezas y el potencial de las personas con discapacidad. Igualmente enfocarse en la persona y no simplemente en los límites de su condición.

Considerando los hallazgos y limitaciones del presente trabajo se recomienda la realización de estudios cualitativos longitudinales con un mayor grupo de participantes. Este diseño permitiría obtener más información sobre el impacto personal y familiar de la inclusión educativa y del acceso a los servicios de salud en personas con DI. En el futuro, se deben implementar evaluaciones nacionales de servicios infantiles, en modo particular del impacto en las familias con niños con discapacidad. A partir de las mismas, recomendar acciones específicas para abordar las barreras que impiden su adecuada inclusión educativa. Además, se debe desarrollar una guía para las evaluaciones de estos servicios llevados a cabo a nivel local o regional con el fin de asegurar de que se tengan en cuenta adecuadamente las necesidades de la población infantil con discapacidad.

\section{REFERENCIAS BIBLIOGRÁFICAS}

1. McGrath RJ, Stransky ML, Cooley WC, Moeschler JB. National profile of children with Down syndrome: disease burden, access to care, and family impact. The Journal of pediatrics. 2011 Oct 31;159(4):535-40.

2. Organización Mundial de la Salud. Genes and chromosomal diseases. Down Syndrome [internet]. Ginebra, Suiza: [citado el 30 de octubre de 2017]. Disponible http://www.who.int/genomics/public/geneticdiseases/e n/index $1 . h t m l$.

3. Ticona EC, et al. Incidencia y patologías asociadas del síndrome de Down en recién nacidos del Hospital Edgardo Rebagliati Martins. Noviembre 2012-octubre 2013. Revista Médica Basadrina. 2015 (1):15-19.

4. Phelps RA, Pinter JD, Lollar DJ, Medlen JG, Bethell CD. Health care needs of children with Down syndrome and impact of health system performance on children and their families. Journal of Developmental \& Behavioral Pediatrics. 2012 Apr 1;33(3):214-20.

5. Davidoff AJ. Insurance for children with special health care needs: patterns of coverage and burden on families to provide adequate insurance. Pediatrics 2004;114:394-403.

6. Van Gameren-Oosterom HB, Fekkes M, Buitendijk SE, Mohangoo AD, Bruil J, Van Wouwe JP. Development, problem behavior, and quality of life in a population based sample of eight-year-old children with Down syndrome. PLoS One. 2011 Jul 21;6(7):e21879.

7. Silverman W. Down syndrome: cognitive phenotype. Developmental Disabilities Research Reviews. 2007 Jan 1;13(3):228-36.

8. Turner S, Alborz A. Academic attainments of children with Down's syndrome: A longitudinal study. British Journal of Educational Psychology. 2003 Dec 1;73(4):563-83.

9. Dykens EM. Psychiatric and behavioral disorders in persons with Down syndrome. Developmental Disabilities Research Reviews. 2007 Jan 1;13(3):2728.

10. Ward RL, Nichols AD, Freedman RI. Uncovering health care inequalities among adults with intellectual and developmental disabilities. Health \& Social Work. 2010 Nov 1;35(4):280-90.

11. Krahn GL, Walker DK, Correa-De-Araujo R. Persons with disabilities as an unrecognized health disparity population. American journal of public health. 2015 Apr;105(S2):S198-206. 12. Raymaker DM, McDonald KE, Ashkenazy E, Gerrity M, Baggs AM, Kripke C, Hourston S, Nicolaidis C. Barriers to healthcare: Instrument development and comparison between autistic adults and adults with and without other disabilities. Autism. 2017 Nov;21(8):972-84.

13. Williamson HJ, Contreras GM, Rodríguez ES, Smith JM, Perkins EA. Health care access for adults with intellectual and developmental disabilities: A scoping review. OTJR: Occupation, Participation and Health. 2017 Oct;37(4):227-36. 
14. Oliver M, Barnes C. Disability studies, disabled people and the struggle for inclusion. British Journal of Sociology of Education. 2010 Sep 1;31(5):547-60. 15. Ali A, Scior K, Ratti V, Strydom A, King M, Hassiotis A. Discrimination and other barriers to accessing health care: perspectives of patients with mild and moderate intellectual disability and their carers. PloS one. 2013 Aug 12;8(8): e70855.

16. Iacono $\mathrm{T}$, Bigby $\mathrm{C}$, Unsworth $\mathrm{C}$, Douglas $\mathrm{J}$, Fitzpatrick P. A systematic review of hospital experiences of people with intellectual disability. BMC health services research. 2014 Oct 25;14(1):505.

17. Boulter E, Rickwood D. Parents' experience of seeking help for children with mental health problems. Advances in Mental Health. 2013 Feb $1 ; 11(2): 131-42$.

18. Byrne B. Qualitative interviewing. Researching society and culture. 2004 Jun 9;2:179-92. 19. Yeo A, Legard R, Keegan J, Ward K, McNaughton Nicholls C, Lewis J. In-depth interviews. Qualitative research practice: A guide for social science students and researchers. 2014;2:177210.

20. Braun V, Clarke V. Using thematic analysis in psychology. Qualitative research in psychology. 2006 Jan

$1 ; 3(2): 77-101$.

21. Pace, J. E., Shin, M., \& Rasmussen, S. A. (2011). Understanding physicians' attitudes toward people with Down Syndrome. American Journal of Medical Genetics Part A, 155, 1258-1263.

22. Gavaldá JM, Qinyi T. Improving the process of inclusive education in children with ASD in mainstream schools. Procedia-Social and Behavioral Sciences. 2012 Jan 1;46:4072-6. 23. Thompson N. Anti-discriminatory practice: Equality, diversity and social justice. Palgrave Macmillan; 2016.

24. Radermacher H, Sonn C, Keys C, Duckett P. Disability and participation: It's about us but still without us!. Journal of Community \& Applied Social Psychology. 2010 Sep 1;20(5):333-46.

25. Fernández Castillo P. ¿Cómo se dan los derechos educativos de los niños, niñas, adolescentes con discapacidad en el Perú?: estudio cualitativo en Huancavelica y Villa El Salvador. 2007. Foro educativo. Lima.

26. Green S, Davis C, Karshmer E, Marsh P, Straight B. Living stigma: The impact of labeling, stereotyping, separation, status loss, and discrimination in the lives of individuals with disabilities and their families. Sociological Inquiry.
2005

May

1;75(2):197-215.

27. Skotko BG, Levine SP, Goldstein R. Having a son or daughter with Down syndrome: Perspectives from mothers and fathers. American Journal of Medical Genetics Part A. 2011 Oct 1;155(10):2335-47. 28. Pelchat D, Levert MJ, Bourgeois-Guérin V. How do mothers and fathers who have a child with a disability describe their adaptation/transformation process?. Journal of Child Health Care. 2009 Sep;13(3):239-59.

29. Andrade RV, Limongi SC. The emergence of expressive communication in the child with Down syndrome. Pró-Fono Revista de Atualização Científica. 2007 Dec;19(4):387-92. 30. Jackson C, Cavenagh P, Clibbens J. Communication and self-esteem in adults with Down syndrome. International journal of language \& communication disorders. 2014 May 1;49(3):275-87. 31. Sigafoos J, O'Reilly M, Green VA. Communication difficulties and the promotion of communication skills. En A. Carr, G. O'Reilly, P. Noonan Walsh and J. McEvoy (eds), The Handbook of Intellectual Disability and Clinical Psychology Practice. Routledge. London; 2010:606-643.

32. Bray M. Is Stuttering Therapy Useful for Adolescents and Adults with Down Syndrome who are Dysfluent?. Procedia-Social and Behavioral Sciences. 2015 Jun 30;193:51-61. 33. Buckley S, Bird G, Sacks B, Archer T. A comparison of mainstream and special education for teenagers with Down syndrome: Implications for parents and teachers. Down Syndrome Research and Practice. 2006 Jan 1;9(3):54-67. 
ISSN 2519-0652 CASUS.2017;2(3):169-178

\section{Impact of health and education systems on the experience of a mother with a child with Down syndrome}

ABSTRACT: Children with Down syndrome have various health, behavioral and learning problems that require special attention. Because of this, they have difficulties in receiving adequate attention. Consequently, inefficiency in the health and education system impacts on a personal and family level. However, in Peru, there is a gap of empirical and qualitative information about the impact on the lives of children with Down syndrome and their parents. Using a qualitative analysis of the contents of an interview with a mother, seven themes linked to the impact of these two areas were identified: orientation and support, work environment and health condition, relationship with the health system, relationship with the school, discrimination towards people with disabilities, change of perspective regarding disability and unconventional communication. The results show that the forms of inefficiency of the health and education systems have a negative impact on the lives of people with disabilities and their families. This impact affects family and work life, it also determines educational and health decisions. Likewise, there are still persistent forms of non-acceptance and more or less evident forms of discrimination, notwithstanding efforts for inclusion. Negative attitudes towards disability can be conditioned by their severity. The acceptance of disability is a process in which perception is modified. In it, it is fundamental to focus on the person and not simply on their limited condition.

KEY WORDS: Intellectual disability; Down syndrome; Family; Health systems; Qualitative analysis; Peru. 